\title{
ANESTHETIC MANAGEMENT IN THE PATIENT WITH GIANT RETROPERITONEAL LIPOSARCOMA: A CASE REPORT
}

\author{
Toleska $\mathrm{M}^{1}$, Kartalov $\mathrm{A}^{1}$, Kuzmanovska $\mathrm{B}^{1}$, Dimitrovski $\mathrm{A}^{1}$, Ognjanova $\mathrm{V}^{1}$, Naumovski $\mathrm{F}^{1}$ \\ ${ }^{1}$ University Clinic for TOARILUC - KARIL, Medical Faculty; University “Ss Cyril and Methodius" Skopje, \\ Republic of Macedonia
}

Background : Liposarcoma is a rare type of malignant tumor of mesenchymal origin. It occurs most commonly in the extremities (52\%), retroperitoneum (19\%) and inguinal region (12\%). Retroperitoneal liposarcoma is usually asymptomatic until the liposarcoma is large enough to compress the surrounding organs. The retroperitoneum is a large space in which the retroperitoneal liposarcoma grow and these tumors are known to reach significant dimensions, despite their poor vascularization and can grow to enormous size. The symptoms would only occur if the liposarcoma presses on the surrounding organs.

Case report : A 66-year-old woman (height $157 \mathrm{~cm}$; weight 80 $\mathrm{kg}$ ) with a giant abdominal tumor was scheduled for tumor resection under general anesthesia (Figure 1). The computed tomography (CT) of the abdomen and pelvis demonstrated large ventral abdominal hernia, which is consisted of gaster, small and large intestine and left kidney, suspect for large abdominal tumor. The preinduction vital signs were: BP: 163/97 mmHg, HR: 68/min, RR: 18/min, $\mathrm{SpO}_{2}=91 \%$. We placed:

- 2 large intravenous canula with cristaloid solution, - premedication with midazolam $1 \mathrm{mg}$ and fentanyl $50 \mu \mathrm{gr}$,

- epidural catheter in the Th11-Th12 space,

- arterial catheter was inserted into the left radial artery,

- central venous catheter was placed in right internal jugular vein (to monitore CVP and to administer vasoactive drugs).

Surgeons found giant tumor mass which was incapsulated and situated intrabdominaly and retroperitonealy (Figure 2) :

- total surgery time was 285 minutes,

- anesthesia time was 360 minutes,

- total blood loss was around $1500 \mathrm{ml}$ and $700 \mathrm{ml}$ of ascites (we administered 4 packages of RBC and 4 packages of plasma).

There were two episodes of decreased ABP and CVP as a result of surgical manipulation with the huge tumor mass, who was compressing the inferior vena cava. The vital parameters remained stable all the time during the surgery.

The intravenous fluid was titrated to maintain CVP between 8-12 $\mathrm{mmHg}$ and IAP below $20 \%$ of the baseline measurement. The dimension of tumor was $56 \times 52 \times 20 \mathrm{~cm}^{3}$ and its weight about 32,9 $\mathrm{kg}$. Complete surgical resection was done and the giant tumor mass was removed together with descending colon, left kidney and left ureter.

\section{References :}

1. Zhang WD, et al. Management of retoperitoneal liposarcoma: A case report and rewiew of the literature. Oncol Lett $2015 \mathrm{Jul} ; 10$ (1): 405-409.

2. Caizzone A, et al. Giant retroperitoneal liposarcoma: case report and rewiew of the literature. Int J Surg Case Rep 2015; 9: 23-26.

3. Feng $\mathrm{D}$, et al. Anesthetic management of a patient with giant retroperitoneal liposarcoma: case report with literature review. Int J Clin Exp Med 2015; 8: 19530-34.
Discussion : Retroperitoneal liposarcoma commonly occurs between 40-60 years of age and has a 1:1 ratio between genders. They grow slowly and silently and may not become symptomatic until they are very large, so diagnosis is often delayed.

Patients with giant retroperitoneal liposarcoma are considered to have great risk of perioperative complications and require precise anesthetic management.

The giant retroperitoneal liposarcoma may :

- increase abdominal pressure,

- compress abdominal aorta and inferior vena cava,

- compress the gastrointestinal tract (anemia or malnutrition),

- the tumor may lift up the diaphragm and cause reduced respiratory compliance and respiratory distress,

- rapid removal of tumor may lead to unexpected redistribution of blood or massive blood loss and that can lead to other complications such as hypotension, anemia, cardiac arrhythmia.

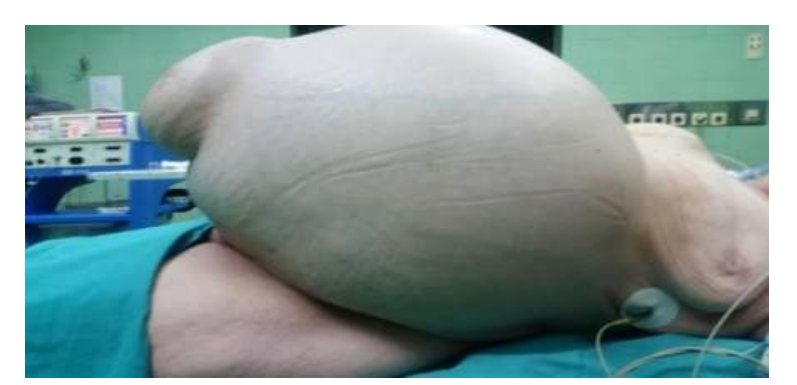

Figure 1: Perioperative view of the patient (showed remarkably distended abdominal tumor).
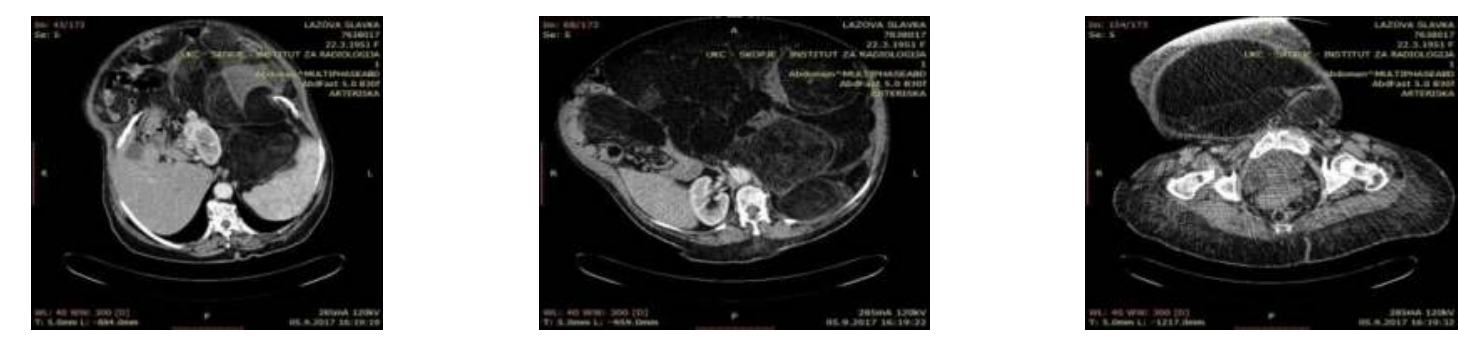

Figure 2: Computed tomography (CT) scen shows incapsulated enormous tumor mass situated intraabdominaly and retroperitonealy (described as large ventral abdominal hernia).

Learning points : Anesthetic management for patients with giant retroperitoneal liposarcoma must be focused on:

1. Perioperative use of invasive monitoring (ABP and CVP) ;

2. Adequate fluid management ;

3. Avoidance of compression on abdominal aorta and inferior vena cava ;

4. Maintenance of adequate venous return ;

5. Prevention of pain ;

6. Hypoxaemia, hypercarbia, acidosis, temperature control ;

7. We must be aware of postoperative complications, especially respiratory distress (because of the giant size of the liposarcoma). 